\title{
Mit Profil in den Wettbewerb
}

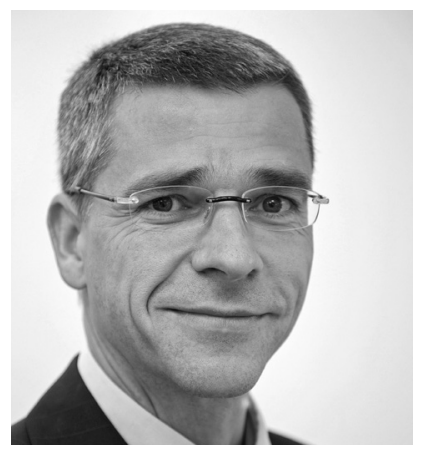

VON THOMAS HILSE

Dr. Thomas Hilse hat nach seinem Studium der Betriebswirtschaft mit Schwerpunkt Marketing und der Promotion im Gesundheitsbereich einer international tätigen Unternehmensberatung sowie im Geschäftsfeld Versorgungsmanagement eines Versicherungsunternehmens gearbeitet. Er ist stellvertretender Vorstandsvorsitzender des Bundesverband Managed Care e. V. und Lehrbeauftragter an der Hochschule Fresenius. www.hilsekonzept.com

\author{
In einem Forum des Kongresses "Klinikmarketing \\ 2012" wurden zahlreiche Praxisbeispiele \\ vorgestellt, die auch auf andere Geschäftsfelder \\ der Sozialwirtschaft anregend wirken können.
}

Marketing wird für soziale Einrichtungen in Zeiten steigenden Wettbewerbs immer bedeutender. Dies betrifft insbesondere für Dienstleister im Geschäftsfeld Senioren und Pflege zu. Über Erfolgskonzepte und Praxisbeispiele, aber auch über bestehende Defizite diskutierten am 5. November 2012 Referenten und Teilnehmer des erstmals auf dem Klinikmarketing-Kongress des Veranstalters rotthaus.com in Köln durchgeführten Forums. Das Kongressforum wurde in Kooperation mit dem Bundesverband privater Anbieter sozialer Dienste e. V. (bpa) durchgeführt.

Am Beispiel »Finkenau «, einer großen Senioreneinrichtung in Hamburg, erläuterte Frank Haesloop, Direktor des zu Pflegen \& Wohnen gehörenden Hauses, die Vorbereitung und Durchführung eines Markteintritts. Die kennzahlengestützte Marktanalyse für den Standort schafft die Grundlage für die Erarbeitung eines eigenständigen Profils. Von besonderer Bedeutung ist seiner Ansicht nach die frühzeitige Auswahl und aktive Einbindung des zukünftigen Managements (Direktor, Pflegedienstleitung). Beide sollten idealerweise den Markt nicht nur generell kennen, sondern auch bei Zuweisern in der Region bekannt sein. Auch müssen das Objekt und das Angebot klare Differenzierungsmöglichkeiten gegenüber dem Wettbewerb ermöglichen. Eine besondere Herausforderung beim Markteintritt seiner Einrichtung stellte die Re-Positionierung des vormals als Niedrigpreisanbieter bekannten Unternehmens Pflegen \& Wohnen dar.

In seinem Referat arbeitete Martin Kaiser, Geschäftsführer der Städtischen
Seniorenheime Dortmund gGmbH, die besondere Lotsenfunktion der Kommunen heraus. Kommunen könnten durch ihre Strukturen insbesondere im Quartier wertvolle Unterstützung für Zuweisung und Vernetzung leisten. Für ihn sind stationäre Pflegeeinrichtungen die eigentlichen Zentren, um die sich alle anderen Partner und Dienstleister herum gruppieren sollten.

Dr. Bernd Schubert, vormaliger Regionalgeschäftsführe bei der AMEOSGruppe und jetziger kaufmännischer Geschäftsführer bei der AWO Schleswig-Holstein, erläuterte in seinem Referat, die Herausforderungen an die Führung kleinerer Einrichtungen in strukturschwachen Regionen. Ein stabiler Kundenzugang ist nur über ein breites und zugleich ausdifferenziertes Angebotsspektrum zu gewährleisten. Gerade in ländlichen Strukturen ist eine tiefe Einbindung in die kommunalpolitischen und vereinsbezogenen Kommunikationsprozesse von hoher Bedeutung. Vergleichbares gilt für die medizinischen Leistungserbringer der Region sowie die Selbsthilfeorganisationen und Selbsthilfegruppen. Als Erfolgsfaktoren für den bestmöglichen Kundenzugang in strukturschwachen Gebieten kennzeichnete der Referent die lokale Bindung, die professionelle Leitung, die Qualität des Angebots, das aufgebaute Vertrauen in die Einrichtung sowie die aktive Einbindung des Ehrenamts.

Als Zuweisermarketing kann die zielgerichtete Beeinflussung von Bewohnerströmen zum Zweck der Auslastungssicherung bezeichnet werden. In seinem Vortrag hob Dr. Michael Os- 
sege, Fachanwalt für Medizinrecht in der Kanzlei Rechtsanwälte Wigge aus Münster, insbesondere die Grenzen des Zuweisermarketing hervor. Gerade bezogen auf ärztliche Zuweisung sind den Handelnden enge Vorgaben durch die Berufsordnung gegeben. So dürfen Ärztinnen und Ärzte für die Zuweisung von Patientinnen und Patienten kein Entgelt oder andere Vorteile fordern. Bei Verstößen drohen nicht unerhebliche Konsequenzen in Bezug auf Approbation oder Zulassung. Unbeschadet dessen sieht aber gerade das SGB V im Rahmen des Versorgungs- und Entlassmanagements sowie der jeweiligen Landesverträge eine enge Kooperation zwischen Ärzten und Pflegeeinrichtungen vor. Insofern, so Ossege, sei Zuweisermarketing im Rahmen des rechtlich Möglichen auf kooperativem Weg ausdrücklich als notwendig zu bejahen.

Der zweite Kongressteil beschäftigte sich mit erfolgreichen MarketingKonzepten aus der Praxis. Unter der Moderation des Autors, in dessen Verantwortung die inhaltliche Gesamtverantwortung des Kongressforums lag, stellten fünf Referenten ihre aktuellen Ansätze vor:

- Marketing - kreativ und wirtschaftlich: Eine ausdrückliche Auseinandersetzung mit den Zielgruppen des Marketing stellte Alexander Dettmann, Geschäftsführer der AGAPLESION BETHANIEN DIAKONIE gGmbH aus Berlin, an den Anfang seiner Ausführungen. Entscheidungen für eine Pflegeeinrichtung sind komplex und durch die aktive Mitwirkung mehrere Personen (Betroffene, Angehörige, Ärzte) gekennzeichnet. Daher müsse Marketing auch früh ansetzen und die relevanten Multiplikatoren einbinden. Trotz des schwierigen Themas »Pflege« sollten Emotionen transportiert werden, die zur Reduzierung der Berührungsängste beitragen sollen.

- Integriertes Zuweisermarketing: Mit der bedarfsgerechten Gestaltung der Leistungen entlang der Versorgungskette zur Sicherung des Kundenzugangs setzte sich Sven-Uwe Gau, Leiter der MediClin Seniorenresidenz Deister Weser, auseinander. Als Alleinstellungsmerkmal hat seine Einrichtung die gerontopsychiatrische Pflege herausgearbeitet und baulich in Form eines Wohngruppenkonzepts umgesetzt. Über eine gezielte Auswahl von Kooperationspartnern unterstützt eine abgestimmte Öffentlichkeitsarbeit die Multiplikatoren und das Netzwerk entlang der Versorgungskette. Dabei wird bewusst zwischen Profis und Laien unterschieden. Soziale Medien spielen nur in Bezug auf die Mitarbeitergewinnung eine Rolle.

- Markenbildung: Heiko Langheim, Geschäftsführender Gesellschafter der Haus am Berg Seniorenpflege und -betreuung $\mathrm{GmbH} \& \mathrm{Co}$. KG in Hasbergen, präsentierte in seinem Vortrag das Herausbilden einer Marke am Beispiel einer kleinen Senioreneinrichtung. Für sein Haus, ein Pflegeheim der vierten Generation im Hausgemeinschaftsmodell, ist Markenbildung ein Teil des persönlichen Marketing-Mix. Nachdem alle Senioreneinrichtungen pflegerische Dienstleistungen anbieten würden, muss im Rahmen der Produktpolitik das Einzigartige herausgearbeitet werden. Pflegesatzpolitik im Sinne der klassischen Preispolitik ist nur bedingt möglich. Die Kommunikationspolitik wird, so der Referent, durch eine Corporate Identity mit einem guten Logo bestimmt, welches durch einen stimmigen Slogan ergänzt werden sollte. Als Grundsätze der Öffentlichkeitsarbeit und Werbung gelten für den Referenten Wahrheit, Klarheit, Wirksamkeit, Wirtschaftlichkeit und Aktualität. Alle Maßnahmen müssten diesen Anforderungen genügen.

- Qualitätsbericht als Marketing-Instrument: Einen besonderen Ansatz zur zielgruppenspezifischen Kommunikation von Senioreneinrichtungen stellte Andreas Lammers vor. Er ist Geschäftsführer der DKV-Residenz am Tibusplatz gGmbH in Münster und verantwortet zudem die DKVResidenz in Bremen sowie zwei ambulante Pflegedienste. Der kundenorientierte Qualitätsbericht seiner beiden Residenzen trägt zur Minderung der Kommunikationslücke zwischen Dienstleistungsanbieter und Nachfrager bei. Er unterstreicht den Wettbewerbsvorteil durch belegbare Qualität. In der Print-Version besteht der Bericht aus einem Magazin-Teil mit Geschichten aus den DKV-Residenzen und illustriert so das tatsächliche Leben in den Einrichtungen.
Ein Tabellenteil gibt ausführliche Antworten auf wesentliche Fragen und ermöglicht so eine Vergleichbarkeit mit Dritten. Die Kombination aus erlebbaren Eindrücken aus dem Tagesgeschäft und der transparenten Darstellung der Fakten kann die Alleinstellung der Einrichtungen unterstützen. Insofern wird dieses Instrument intensiv und erfolgreich für Kundenakquise, Kundenbindung und Kundenkommunikation sowie für Multiplikatoren eingesetzt. Es ist auch online verfügbar.

- Personalmarketing: Am Projektbeispiel eine Sozialstation zeigte Dr. Maren Kentgens, Managerin von Lischke Consulting aus Hamburg, die Herausforderung einer langfristigen Mitarbeiterbindung und Mitarbeitergewinnung auf. Dazu definierte sie zunächst das Erfordernis eines Dreiklangs aus Leistungsprofil, Attraktivität als Arbeitgeber und Prozesse, um die Komplexität des Personalmarketings zu strukturieren. Die Rolle des Arbeitgebers in diesem Thema ist wesentlich durch sein Image geprägt und ergibt sich aus der systematischen Bearbeitung zahlreicher interner und externer Kontaktpunkte. Unter aktiver Einbindung der Mitarbeiter, so die für die Projektdurchführung verantwortliche Beraterin Susann Pöwe, konnten konkrete Maßnahmen für Personalgewinnung und Personalbindung erarbeitet werden. Die Attraktivität des Arbeitgebers ist ein wesentliches Erfolgsrezept gegen den Fachkräftemangel.

\section{Fazit}

Marketing für Senioreneinrichtungen trägt maßgeblich zur Differenzierung im Wettbewerb bei. Dabei sind von den Häusern die klassischen MarketingInstrumente einschließlich einer stringenten Erfolgskontrolle einzusetzen. Ein bedeutsamer Multiplikator in Richtung der spezifischen Zielgruppen sind die Mitarbeiter der Einrichtungen. Sie tragen wesentlich zur Imagebildung bei Pflegebedürftigen und ihren Angehörigen bei. Eine authentische und ehrliche Kommunikationspolitik kann darüber hinaus einen hohen Beitrag zur weiteren Professionalisierung und zur Imageverbesserung der gesamten Pflegebranche leisten. 